\title{
Safety Critical Incidents Among Small Construction Contractors: A Prospective Case Study
}

\author{
Tonya Smith-Jackson*,1, Sharnnia Artis ${ }^{2}$, Yu-Hsiu Hung ${ }^{1}$, Hyung Nam Kim ${ }^{1}$, Caleb Hughes ${ }^{3}$, Brian \\ Kleiner $^{1}$ and Alicia Nolden ${ }^{1}$
}

\author{
${ }^{I}$ Virginia Polytechnic Institute and State University, Center for Innovation in Construction Safety and Health Research, \\ 250 Durham Hall, MC0118, Blacksburg, VA, 24061, USA \\ ${ }^{2}$ Sharnnia Artis, Human Factors Engineer, Aptima, Inc., 3100 Presidential Drive, Suite 220, Fairborn, OH 45324, USA \\ ${ }^{3}$ Caleb Hughes, System Safety Engineer, Systems Engineering \& Advanced Technology Division, Science Applications \\ International Corporation, 100 Greenspring Dr., Stafford, VA 22554-1705, USA
}

\begin{abstract}
According to a 2006 Bureau of Labor Statistics (BLS) report, approximately $90 \%$ of construction companies employ 20 or fewer workers; of these, $30 \%$ specialize in residential construction. Contractors with 10 or fewer employees are exempt from OSHA reporting requirements, unless a fatality occurs or an injury/illness leads to lost workdays for three or more employees (29 CFR 1904.1, Subpart B). This qualitative case study was conducted to understand characteristics and work patterns to develop a clearer understanding of the natural context of work, especially given the underreporting of safety information by small construction companies. A prospective study was conducted in which construction workers logged critical incidents for four weeks. Definitions and examples of critical incidents were given to workers, along with a data logging notebook with structured questions. Workers could report the events immediately upon occurrence or after the workday ended. A total of 102 critical incidents were reported. Content (axial coding) and cluster analyses were used to analyze the logs and to extract important descriptors and patterns. Results were used to characterize injury types, antecedents, time-of-day, and family-work involvement. Descriptions regarding the training-, resource-, and planning-related practices of small construction workers focused on the unique challenges that impose barriers and constraints on safety climate.
\end{abstract}

Keywords: Safety, small construction, critical incidents.

\section{INTRODUCTION}

Small contractors present a significant challenge to occupational health and safety. Due to their size, limited resources, and unique stressors related to work-family conflict and transient workers, this portion of the construction industry is difficult to monitor and, at times, exempt from certain Occupational Safety and Health Administration (OSHA) regulations. In the USA, $90 \%$ of all construction companies employed fewer than 20 workers. Of those, $65 \%$ employed 5 or fewer workers [1]. OSHA requires companies with fewer than 11 workers to participate in reporting or monitoring of injuries only if there is a fatality, or if a single accident leads to injury of three or more workers plus lost workdays (29 CFR 1904.1, Subpart B). Thus, these companies operate below the awareness of most of the spectrum of safety and health regulators and researchers. Research substantiates the association between company size and safety performance [2]. Small construction companies had more safety problems compared to larger construction companies, while management and employee commitments also trended lower compared to larger construction companies.

*Address correspondence to this author at the Virginia Polytechnic Institute and State University, Center for Innovation in Construction Safety and Health Research, 250 Durham Hall, MC0118, Blacksburg, VA, 24061, USA; Tel: 540-231-4119; Fax: 540-231-3322:

E-mail: smithjack@vt.edu
A similar problem exists internationally and seemingly on a much larger scale. Most construction companies outside of the USA are considered informal, having little formal structure and organization, but providing very important contributions to the overall economies of developing countries [3]. Likewise, the smaller companies are more likely to hire migrant workers, some of which are undocumented [4]. For example, in the USA, a large proportion of workers from Mexico and Central America are employed in the agriculture and small construction companies, both of which have a disproportionate amount of fatalities compared to all other occupational sectors [5]. In Taiwan, Filipino migrant workers, who are concentrated in small construction and agricultural companies, experience a significant number of illnesses related to exposures to toxic substances [3]. In Tanzania, migrants from surrounding countries show the same pattern [3], as well as workers from countries such as India, Turkey, Pakistan, and Bangladesh. This phenomenon alone supports the urgent need to conduct comprehensive safety and health research that targets smaller companies.

\subsection{Safety Challenges for Small Construction Contrac- tors}

Small contractors tend to inherit the problems that were not resolved in the earlier construction planning stages. The early work done by designers, planners, and larger contractors make it difficult for small contractors to provide inputs 
to ensure contract obligations are more compatible with their resource constraints and capabilities (i.e., scheduling). Garber [6] describes the shifting of liability and risk to small contractors and subcontractors as the "construction food chain", which refers to the transfer of risk from larger contractors to smaller contractors and to subcontractors. Similar to the common system development cycle problems, early planning, conceptual design, and development work that does not conform well to safety and health objectives make it difficult for downstream developers (such as the product designers and evaluators) to make the final product safe and usable.

As an example, 47 subcontractors were surveyed to determine risks associated with the role of subcontracting [7]. Three work groups were sampled; mechanical (e.g., ventilation, fire protection), licensed (e.g., plumbing, electrical), and ordinary (e.g., semi-skilled or unskilled trades). Several factors were identified that subcontractors associated with increased risk of injuries. For example, subcontractors reported that contracts contain requirements and conditions that are unfavorable in terms of time extensions and payment. About $66 \%$ of the subcontractors perceived subcontract conditions to be unfair, and the smaller the subcontractor the stronger the perception that contract terms and conditions interfered with safety. Work design to adapt to these constraints places workers at a higher risk of injuries or fatalities.

Mayhew and Quinlan [8] also found a similar perception in Australia and the United Kingdom. After conducting longitudinal surveys over a 5-year period, researchers found a strong link between self-employed builders and chronic injury. This study also identified a pattern of transfer of both financial and health and safety risks from larger contractors and subcontractors to smaller subcontractors. Four key factors were identified that explain the relationship between health and safety risks and outsourcing. These were: (1) Economic/reward pressure; (2) Disorganization; (3) Diminished regulation; and (4) Inability of outsourced labor to organize.

Unlike larger contractors, small contractors do not have many resources to mitigate the hazards they encounter in construction. They face additional stressors not found in the larger construction entities. These additional stressors include organizational design, challenges to participation in business development, and work family conflict. Small contractors do not establish formalized operations, typically. Logically, their small size may not always require a formalized work system to support role assignments and communication chains. So, small contractors function without formal policies and procedures, although some values and climate factors may be implicit. There are often unspoken expectations for workers' behaviors and practices.

Unfortunately, the expectations are self-developed, and are often not learned from best practices gained through business development and networking. Small contractors are less likely to collaborate with similar contractors, and are reluctant to share best practices or other helpful information [9]. Moreover, these businesses resist participating in strategic business networks unless there is a direct positive benefit to the bottom line [10]. Although some family-owned firms enjoy the benefits of family ties in the workplace, these firms tend to be overcome by work and family conflict [11, 12], which increases the risks of hazard exposure.

Nonetheless, construction businesses are the most favorable to self-employment ventures [1] when compared to other industry types, including agriculture and service industries. Benefits include higher job satisfaction compared to medium and large contractors [13], and workers have a more positive view of the supportiveness of supervisors compared to larger contractors, and perceive their work environments are flexible, innovative, and family-oriented [14-16].

\subsection{Safety Culture}

Safety culture and safety climate are difficult to assess in small organizations. The lack of knowledge about safety culture in relatively informal work systems is a major impediment to the development of prevention and control measures that target this population of workers who are over-represented in the injury and fatality data. Safety culture affects workers' attitudes and behaviors [17]. A holistic view of the organization is essential to implementing effective prevention and control measures. Without an understanding of safety culture, it is difficult to determine which prevention and control measures are absent or ineffective, and which areas of performance require more attention (i.e., training). In contrast, safety climate of informal work systems may be easier to assess. In a comprehensive literature review by Wiegmann, Zhang, von Thaden, Sharma, and Mitchell [18], safety climate was often described as a surface feature of the more abstract safety culture. The consensus view describes safety climate as "a temporal phenomenon, a snap shot of safety culture that is relatively unstable and subject to change (p. 8)." These snap shots may take the form of critical incidents, which reflect temporary states that, as a whole, may be used to draw inferences about safety culture. One goal of this study was to identify critical incidents elicited by the safety climate and decompose them into specific attributes that may, in the long term, serve as a surface-level description of safety culture in small, informal construction work systems. While workers from different businesses participated in the study, it was our goal to examine safety climate at the individual level and not at the group level. Critical incidents serve as a mechanism to identify in situ events that provide a snap shot of the organization.

\subsection{Relevance of the Critical Incident Technique}

The Critical Incident Technique (CIT) is a useful method to acquire experienced-based information about environments such as construction work systems. The CIT was first developed by Flanagan [19] for use in aviation and combat leadership. Flanagan defined the method broadly as a "set of procedures for collecting direct observations of human behavior in such a way as to facilitate their potential usefulness in solving practical problems.... (p.1)." The method supports real-time observation of events and the use of descriptions of the events to develop an understanding of system-critical features that require further attention in terms of design or evaluation. Participants simply tell a story or describe an event. Critical incident data provide a number of opportunities to gain a comprehensive understanding of the context of a work system and the worker's experience. 
Up to this point, the extant literature shows no empirical research on the application of the CIT to understand construction work systems, with the exception of De Saram, Ahmed, and Anson [20]. De Saram et al. used the CIT to assess the quality of project managers' coordination of construction projects. The primary goal was to determine whether the CIT was a useful method to measure construction coordination quality. Participants were interviewed and scenarios were used to elicit CI reports. Thirty-six CIs were collected from 21 project managers. These incidents were then categorized into a meaningful hierarchical taxonomy. The results were then interpreted and researchers were able to identify directly observable (explicit) factors related to quality, and were able to draw inferences to identify implicit factors. De Saram et al. highlighted the advantages and relevance of the CIT in construction work systems. These advantages included support for the identification of input, process, and outcome concepts; ease of application to multiple stakeholders; extraction of constructs that reflect the informal and intangible aspects of work systems; and generation of results that can be used immediately to improve the system.

\subsection{Purpose of the Study}

This study used a prospective case study approach to acquire self-reported critical incidents from workers in small construction companies. The prospective approach was selected to minimize confounds associated with memory and recall. The primary purpose was to explore the small construction work context based on the self-reported critical incidents of workers. Two main objectives were pursued:

1. To understand the types of CIs occurring in small construction subcontracting.

2. To describe patterns of CIs emerging from characteristics of the work context (e.g. time of day).

A qualitative approach was used, because the purpose of the study was to illuminate and describe what is happening in the natural construction work environment and not to test pre-set hypotheses. In qualitative studies, the goal is to explore complex psychosocial phenomena such as behaviors and attitudes in the natural context rather than isolating and reducing phenomena to smaller, measurable elements [21].

\section{METHOD}

\subsection{Participants}

Although the initial sample consisted of 16 participants, two cases were dropped due to missing data. The two participants with missing data were unable to complete the study because they resigned from their companies and relocated. Since we used follow-up phone calls to corroborate the reports, this step could not be done because the two participants could not be reached. Thus, a total of 14 workers from 8 different companies comprised the sample; 2 females and 12 males. The construction occupations and trades represented were supervisor/foreman, carpentry, roofing, flooring, transportation, and 'general', which described workers who did not specialize in a particular job. The median age was 36 (range $=19$ years to 51 years). Company sizes ranged from 5 to 20 employees, with a median of 5 employees. The median number of years with the current company was 6 .

\subsection{Questionnaires}

\section{Demographics and Safety Climate}

A short demographic questionnaire was administered to each participant to elicit information about age, trade, and company size. The questionnaire contained three additional items to assess safety climate. As stated, our goal was to minimize participants' time to complete initial questionnaires and CI logs due to the time constraints and other pressures characteristic of small construction companies. Two constructs were selected and scaled using a $1-10$ rating job satisfaction and perceived vulnerability. Job satisfaction was assessed using the question "How satisfied are you in your current job?" Perceived vulnerability was assessed using the question "How much control do you feel you have over what happens to your safety on the job?" [see 18, 22, 23]. A third item elicited the number of accidents the worker had experienced while working in construction.

\section{Critical Incident Reporting}

A critical incident log was developed and provided to participants in a notebook. Critical incidents were defined as: “.... any event that is believed to be a significant indication of a system problem, in your case, a safety or health related problem."We further defined a CI a second time using less technical language as:

“...any event, minor or major, where there is a potential risk of injury. A slip, minor cut, loss of balance, fall, or anything that can lead to one of these events can all be considered as critical incidents."

The log design was based on Flanagan's requirements for valid collection of CI data. For example, according to Flanagan, a scenario or definition should be provided during the familiarization session before participants begin logging data. In addition, questions should only elicit the situations observed and the effects of the situations on the constructs of interest (i.e., safety). The questionnaire should encourage the participants to record the events as soon as possible, and participants should be given instructions on how to enhance the accuracy of recall if the CI could not be recorded immediately. The logs also included a list of structured questions to answer when a critical incident occurred. The order of the questions supported a cued recall structure that elicited information in a deductive process (from general to specific). A second level of structure was imposed that included an extended clarification of each question. This second level was used to facilitate consistency in interpretation across workers, while preventing bias among the workers by overclarifying. Table 1 contains the questions, the second-level clarifiers, and the expected variable outcomes for each.

\subsection{Procedure}

The study design was naturalistic and used the self-report method to acquire CIs occurring in the work context (construction work sites). Participants were recruited using contacts in the Center for Innovation in Construction Safety and Health Research (CICSHR) as well as advertisements and word of mouth. Purposeful sampling with minimum variation [24] was used to select participants, using the following criteria to select a relatively homogeneous sample in terms of business size and stability. 
Table 1. Description of CI Log Components (in Order)

\begin{tabular}{|c|c|c|}
\hline Question & Second-level Clarifier & Expected Data Outcome \\
\hline Where did the critical incident happen? & $\begin{array}{l}\text { Was the CI away from the job site, on the job site, above } \\
\text { ground level (on a ladder), in an enclosed area? }\end{array}$ & On-site location patterns/context \\
\hline Describe what happened. & $\begin{array}{l}\text { From what you experienced, explain what events occurred } \\
\text { that led to the CI? }\end{array}$ & Event sequence \\
\hline Describe the task that was being performed. & $\begin{array}{l}\text { What were you or the workers doing at the time the CI } \\
\text { occurred? Try to be as specific as possible. }\end{array}$ & Task and activity types* \\
\hline $\begin{array}{l}\text { Were any family members or friends working } \\
\text { with you involved in this incident? If yes, } \\
\text { please explain your relationship with the peo- } \\
\text { ple involved and their role in the incident. }\end{array}$ & $\begin{array}{l}\text { Were you working with any family members or friends } \\
\text { when the CI happened? If yes, please mention your rela- } \\
\text { tionship with the people involved. What was their in- } \\
\text { volvement in the work activity during the incident? If no, } \\
\text { please mention that you were working alone. }\end{array}$ & $\begin{array}{l}\text { Interpersonal involvement and influence } \\
\text { (family-work conflict) }\end{array}$ \\
\hline $\begin{array}{l}\text { What are some safety and health problems you } \\
\text { see as a result of the critical incident? }\end{array}$ & $\begin{array}{l}\text { What is the extent of harm caused by this CI? Please ex- } \\
\text { plain any possible health risks associated with this CI. (Ex. } \\
\text { I inhaled a lot of toxins while painting that could cause } \\
\text { breathing problems.) }\end{array}$ & $\begin{array}{l}\text { Safety and health consequences (injury } \\
\text { types)* }\end{array}$ \\
\hline $\begin{array}{l}\text { What other factors were involved in causing } \\
\text { the critical incident? }\end{array}$ & $\begin{array}{l}\text { Are there any other factors you have not mentioned? (Ex: } \\
\text { Worker involved is having trouble at home, or is under a } \\
\text { lot of stress). }\end{array}$ & Proximal factors or antecedents* \\
\hline $\begin{array}{l}\text { What are some suggestions you have for pre- } \\
\text { vention of similar incidents in the future? }\end{array}$ & $\begin{array}{l}\text { Is there any way the task could be made safer or is there a } \\
\text { way to decrease the chance that a similar CI will happen? }\end{array}$ & Prevention recommendations \\
\hline
\end{tabular}

*Included in subsequent cluster analysis.

1. Worked in a family-owned construction business with at least 5 and no more than 20 employees.

2. At least 18 years of age.

3. Reachable using a residential phone number.

4. Able to read and write (self-selection will occur, since the advertisements will highlight the requirement of keeping a $\log$ ).

5. Had at least 1 year of work experience with a familyowned construction business.

6. Located in either Virginia or North Carolina.

7. Business must have operated continuously for a minimum of 5 years.

8. Had to be related to the owner/manager or supervisor in a small construction business (or the actual owner/manager may be a participant, but must be related to at least one other employee). This criterion was an attempt to ensure the sample represented the real-world attributes of small construction companies.

9. Willing to attend a training session held in a location of the worker's choosing.

10. Willing to participate in the study for one full month.

11. Able to commit to maintaining confidentiality of the study for the full length of the logging session (28-30 days).
Once workers contacted our researchers, an appointment was arranged to discuss the details of the project, and discuss the meaning of a CI. Informed consent was acquired verbally over the phone, followed by a written document sent through the U.S. postal service. The demographic questionnaire was also completed at that time.

Four logs were given to each worker, and a participant number was recorded on each log. The logs were mailed in a return, self-addressed stamped envelope provided to each worker. Logs were mailed at the end of each 7-8 day period. The mailing schedules were different for each worker based upon their beginning date. The reporting logs were designed to hold up to 70 critical incidents.

Once the informed consent document was signed and returned, the notebook was mailed to the worker followed by a phone call to verify receipt. Although one of the inclusion criteria was a willingness to meet at a convenient location, workers were given the option to complete their familiarization session over the phone for their own convenience. Some of the factors that led to the elimination of this criterion included workers' difficulties with scheduling due to long work hours, having two jobs, and family demands. Additional instructions were given over the phone, namely a review of the definition and suggested methods to report the incidents. Workers were asked to report each critical incident in a discrete manner and were encouraged to record the incident as soon as possible after the occurrence. Follow-up calls were made to each worker during the reporting period to 
remind them to log data and to ensure they returned the notebooks at the end of the reporting period. Workers reported critical incidents for four weeks, and were compensated $\$ 150$ upon completion of the four-week study.

\subsection{Data Analysis}

Phone calls were used to corroborate all reports provided by each participant $[21,25]$. Two methods were used to analyze the data and were aligned with the aforementioned objectives. Content analysis was used to extract characteristics of critical incidents. Cluster analysis was used to identify emergent patterns based upon worker characteristics, the work context, and types of CIs.

CI logs were typed into a text document as they were submitted. Logs were then content-analyzed by using the axial coding method. Preset codes were derived from the structured questions. For example, codes such as time of day and location of incident were recorded by frequency of occurrence, which could then serve as a weighting factor in subsequent analyses. Two independent coders with experience in content analysis methods analyzed the CI log content. Coders were given the definitions of the preset codes. When the training session was completed, the coders analyzed the logs independently. When this step was completed, coders met to compare the codes and reach a consensus on conflicting interpretations. Exact agreement was not difficult to achieve because the incident log was designed to elicit responses in a structured way. For example, codes such as time of day, type of task, or location where the incident happened were clearly articulated by participants. The main areas of disagreement related to incidents that could not be categorized easily. Those incidents were resolved by placing them in the category of "other".

Cluster analysis is an appropriate method to identify patterns or profiles in exploratory data, especially when data mining is needed for data sets that vary by data types and numbers of observations for each element. The method is ideal for data types that include nominal/categorical data. In addition to its validity in identifying patterns within recorded data, the sample sizes used in cluster analysis do not need to conform to the assumptions associated with the Central Limit Theorem; thus there is no rule of thumb for sample size.

SAS $^{\mathrm{TM}} 9.2$ was used to identify patterns within different attributes of the CIs using Proc Cluster. Cluster analysis is useful in identifying observations that form a pattern based on a matrix formed from the raw data set. Themes using content analysis were developed by an independent coder who assigned themes and frequencies to the CI variables, specifically the injury types, results of the CI, tasks performed relevant to the CI, and prevention recommendations. These variables served as the raw data to calculate the distance matrix. Although there is no established rule of thumb for sample size when using cluster analysis, we chose to be cautious in the choice of algorithms for this data set because of the small sample size. For these reasons, a monothetic hierarchical clustering method was used based on the single linkage nearest neighbor method, which also required the use of a small number of clusters $(\mathrm{K})$ and the k-means method for the distance calculations. The monothetic method supports clustering based on one attribute in order to assess simple pat- terns in the data [24]; thus one variable is considered in individual steps.

\section{RESULTS}

\subsection{Characteristics of CIs}

A total of 102 critical incidents were identified and the raw data are summarized here. Forty-seven percent $(47 \%)$ of the critical incidents resulted in minor injuries. Note that workers did not always complete the entire list of questions when logging each critical incident, and workers responded to some questions with more than one response. In some cases, workers would skip a day as well as give more than one response for some of the questions in the log. For example, some workers attributed several factors as causes of a single accident.

\section{Location of Critical Incidents}

Almost all of the CIs occurred on the job site (98; 3 were unreported). One critical incident occurred when a worker was traveling between job sites to deliver materials.

\section{Time of Day}

Many critical incidents were reported between 9 and 11 am and 2 and 4 pm (Fig. 1).

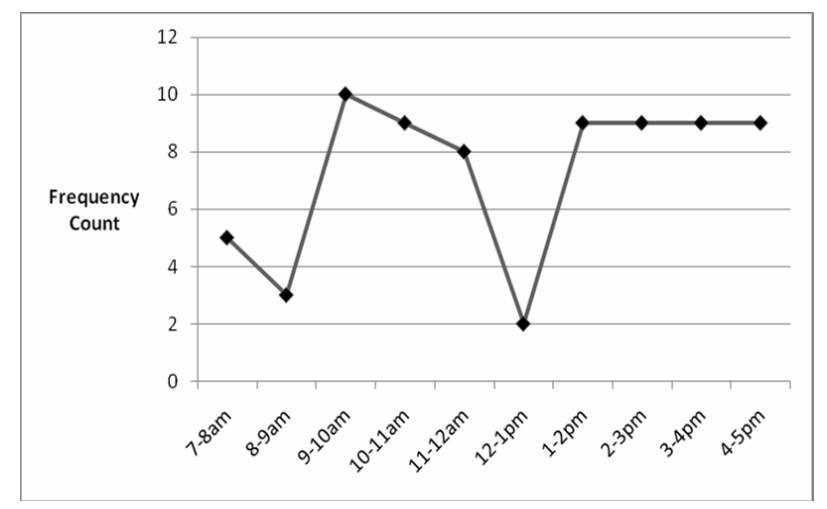

Fig. (1). Critical incidents reported by time of day.

\section{Critical Incident Types}

Trips and falls were the most frequently-reported critical incidents, followed by puncture and laceration injuries usually involving the use of tools. The "other" category consisted of exposure to harmful chemicals, electric shocks, and getting snagged on protruding objects (Fig. 2). Eighty-one different tasks were being performed when the critical incidents occurred. The most common tasks were tool usage $(31 \%)$, working from heights $(27 \%)$, and lifting/carrying loads $(17 \%)$.

\section{Causes of Critical Incidents (Attributions) and Workers' Prevention Recommendations}

Workers were asked to identify the causes or contributors (Fig. 3). The "other" category consisted of medical problems, clutter, outdated or malfunctioning equipment, and improper clothing/jewelry.

Prevention recommendations were also elicited (Fig. 4). The most common suggestions were to wear the proper personal protective equipment (PPE) and attention and awareness of surroundings. 


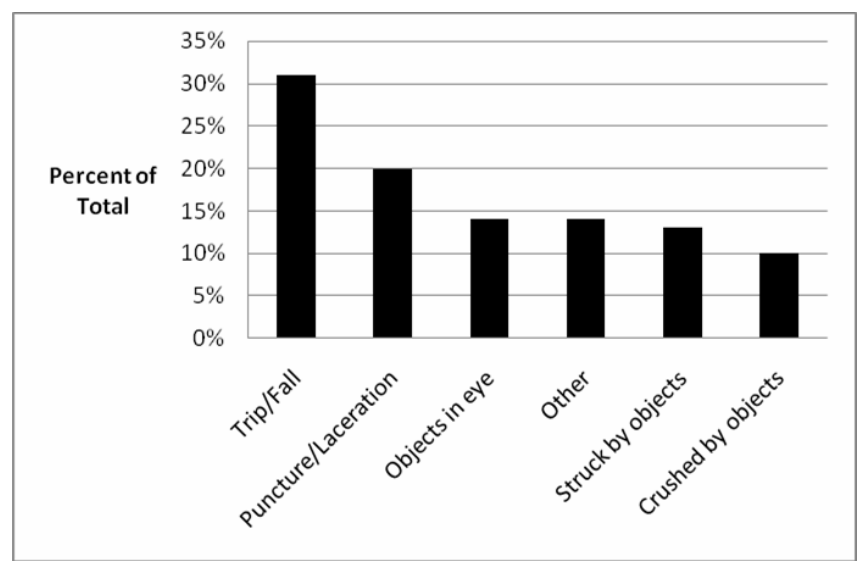

Fig. (2). Critical incidents categorized by type.

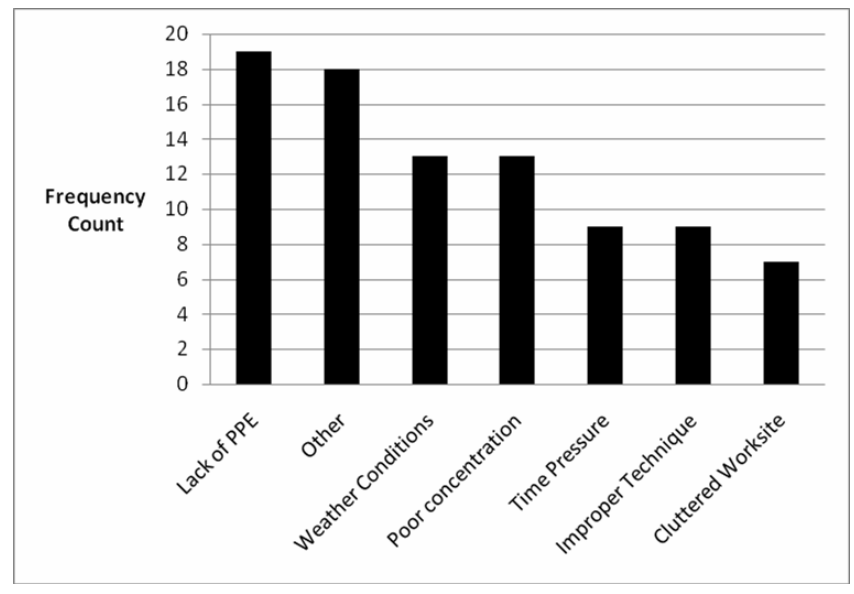

Fig. (3). Workers' attributions of the contributors to critical incidents.

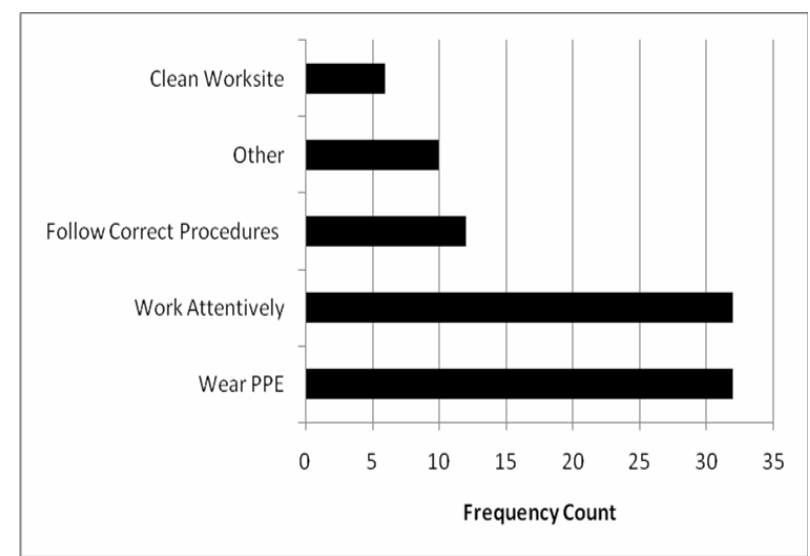

Fig. (4). Workers' recommendations for prevention.

\section{Family-Work Conflict}

A focus on small construction companies provided the opportunity to examine family-work conflict, since many small companies employ family members and friends or acquaintances. Workers were asked to indicate whether a task-related CI was related to the behavior of a friend or family member who was present at the time the CI occurred. Twenty percent (20\%) of the CIs involved family and/or friends.

\section{Safety Climate Construct Correlations}

Spearman-rho correlations were conducted on the three safety climate indicators (ratings); perceived vulnerability, number of accidents in the past year, and job satisfaction. Perceived vulnerability (likelihood of injury) was positively correlated with the number of accidents reported in the past year, $r_{s}(12)=.60, p<.05$. Workers reporting a higher number of past accidents tended to report a higher likelihood that they would be injured on the job. No other significant correlations were identified.

\subsection{Clusters of CIs}

The interpretation of clusters was based on the use of the minimum agglomerative distance, the pseudoFstatistic, and the pseudot ${ }^{2}$. Minimum agglomerative distance algorithms combine individual items into larger categories until all items have been categorized. The pseudoF and pseudot ${ }^{2}$ statistics provide a numerical description of the tightness or distinctiveness of the clusters. Our goal was to identify the smallest reliable cluster solution to provide a preliminary description of the data obtained from the CI logs. A smaller agglomerative distance indicates a stronger cluster solution based upon minimal distance between observations. The variable "injury type" was used in the first cluster analysis. The cluster results for injury type are shown in Table 2. Although an initial eight-cluster solution could be supported, a six-cluster solution was more strongly supported by the combination of the statistics. The minimum agglomerative distance for the six-cluster solution was .13 compared to other minimal distances ranging from .25 to .76 . The pseu$d o F$ value for the six-cluster solution was 1480 compared to the remaining $p s e u d o F$ values ranging from 16.2 to 220 . The pseudot $^{2}$ value was 116 compared to a remaining range of 10.2 to 58.8 . Table 2 summarizes the clusters for the sixcluster solution.

Workers also recorded other factors or conditions they believed contributed to the critical incident. These factors were labeled as "antecedents" and entered as a variable in the cluster algorithm. The same method of thematic assignment was applied by the independent coder. Based on the cluster analysis results, a five-cluster solution was retained. The minimum agglomerative distance of .16 (range $=.16$ to .94 ), a pseudoF value of 44.00 (range $=8.8$ to 248 ), and a pseudot ${ }^{2}$ value of 16.2 (range $=8.8$ to 13.6). Although the pseudoF was not the highest value selected, the selection was based upon agreement with the pseudot ${ }^{2}$ values and the agglomerative distances for the five-cluster solution. Table $\mathbf{3}$ summarizes the elements of each cluster and an interpretation based on similarity.

To determine the types of task patterns that were occurring during the reported CIs, we conducted another cluster analysis using "task type" as the variable. The most reliable solution consisted of five clusters, with agglomerative minimal distance of .29 (range $=.10-.78)$, pseudoF statistic of 104 (range $=54.6$ to 563), and pseudot ${ }^{2}$ statistic of 26.7 (range $=5-54.6$ ). In this case, we gave priority to the simplest solution values to make the cluster selection. Table 4 summarizes the elements of each cluster and an interpretation based on similarity. 
Table 2. Six-Cluster Solution Describing Patterns of Injury Sources

\begin{tabular}{|c|c|c|}
\hline Cluster number & Types of Injury Sources & Label \\
\hline 1 & $\begin{array}{l}\text { Machine damage, combustion exposure/engine fumes, weather } \\
\text { exposure, inhalation of particles, shock }\end{array}$ & Failure to control near-environment hazards. \\
\hline 2 & Cluster 1 plus chemical exposure & See cluster $1^{* *}$ \\
\hline 3 & $\begin{array}{l}\text { Vehicle-related injuries, chemical exposure, crushing of body parts, } \\
\text { machine damage, combustion exposure/engine fumes }\end{array}$ & $\begin{array}{l}\text { Injuries related to operation of machine hazards or outputs } \\
\text { from machines. }\end{array}$ \\
\hline 5 & $\begin{array}{l}\text { Shock, snagged clothing, unspecified, crushing of body parts, ob- } \\
\text { jects in eye, struck by, puncture/laceration }\end{array}$ & Injuries related to distraction or improper techniques. \\
\hline 6 & $\begin{array}{c}\text { Chemical exposure, objects in eye, struck by, puncture/laceration, } \\
\text { trip/fall }\end{array}$ & See cluster $5^{* *}$ \\
\hline
\end{tabular}

*Unspecified injuries consisted of log entries reporting that an injury occurred but not the type of injury. **The cluster includes the previous clustered items PLUS the additional items shown in column 2 of the table.

Table 3. Fiveclusters Describing Patternsof Antecedents*

\begin{tabular}{|c|c|c|}
\hline Cluster number & Antecedents* & Profile Label \\
\hline \hline 1 & Distraction by others, poor visibility, trip/fall hazards, weather, work environment & Safety Climate \\
\hline 2 & Clutter, improper techniques, poor visibility, trip/fall hazards & Safety Climate \\
\hline 3 & Low situation awareness, time constraints & Stress \\
\hline 4 & Faulty equipment, weather & Resources \\
\hline 5 & Lack of safety gear, low situation awareness, weather & Rnd safety climate \\
\hline
\end{tabular}

*Medical conditions and faulty/outdated equipment distances were outliers, thus were not included in the cluster solutions.

Table 4. Five-Clusters Describing Patterns of Tasks

\begin{tabular}{|c|c|c|}
\hline Cluster number & Task Groupings & Working from heights, tool usage \\
\hline \hline 1 & Cleaning, concrete demolition, disengaging machines, vehicle fueling, chemi- \\
cal handling & Roofing tasks \\
\hline 3 & $\begin{array}{c}\text { Disengaging machines, machine/vehicle usage, chemical handling PLUS clus- } \\
\text { ter } 2 *\end{array}$ & See cluster 2 \\
\hline 4 & Cleaning, concrete demolition, disengaging machines, vehicle fueling, chemi- & Mal handling \\
\hline 5 & Lifting/carrying, chemical handling, tool usage & Material handling, tools \\
\hline
\end{tabular}

*The cluster includes the previous clustered items PLUS the additional items shown in column 2 of the table.

\section{DISCUSSION AND CONCLUSIONS}

The first objective of this study was to understand the types of CIs that occur within small construction companies. The CI logs provided a rich data set to conduct analyses that provide a level of examination that is not characteristic of studies of small construction companies. Likewise, the incidents collected in this prospective study provide descriptions of the events occurring within the ecology or naturalistic setting of small construction companies. Descriptive data specific to the on-site challenges of small construction com- panies and patterns that characterize the work environment have not been a focus of empirical research. This study attempted to provide a more detailed snapshot of the small construction work system from the perspective of workers when experiencing CIs, rather than external observers. Trips and falls as well as struck-by and crushed-by injuries were among the highest incident categories, which is consistent with the BLS injury and fatality rankings [1]. This consistency between the critical incidents and national data on fatalities is a preliminary indication of the usefulness of critical incident reporting, since critical incidents can be used as 
early warning signs of potentially more severe events and consequences.

We were also able to acquire anecdotal reports on workers' perceptions of contributors to CIs. Based on the incidents reported here; lack of PPE was mentioned frequently as a cause of specific incidents. Access to PPE is a common complaint among workers in small companies in both construction and agriculture [e.g., 26, 27]. Thus, targeted interventions that focus on low-cost yet effective PPE and demonstrations of the returns-on-investment in terms of safety and lost work days would be of benefit to small contractors. The prevalence of contributors such as weather conditions, distraction, and time pressure is consistent with several prior studies that characterized the barriers to hazard prevention and control within small construction contractors [9, 15, 28]. Small contractors tend to work under conditions of time pressure and task overload. These task pressures are further extended when the work must continue in spite of weather conditions in an attempt to use a small work system to keep up with contract demands and avoid late penalties.

A second objective of the study was to explore behavioral patterns of small construction companies that can be used to support future studies in terms of variable selection, questionnaire development, and observational studies. The patterns may also be useful in creating 2- and 3-dimensional taxonomies to support planning and resource allocations in construction safety projects for subcontractors. In terms of injury types, two important clusters were related to lack of hazard control methods associated with working in the nearenvironment, problems with machine operation, and worker distraction. These types of injuries relate to the need to use administrative controls such as training and work policies. However, the use of training and establishing formal work policies are not common practices among small, informal companies due to time and human resource constraints. In a sample of 818 firms, Kotey\&Folker [29] found significant main effects of company size on the use of informal training vs. formal training. Not surprisingly, smaller companies were less likely to use formal training methods. Informal training may be just as effective; however more scientific studies have focused on effectiveness of certain formal training methods. Unfortunately small business owners do not tend to participate in train-the-trainer opportunities unless there is a direct positive benefit [10].

The patterns of antecedents overlapped due to similarities between clusters. For example, many of the clusters were associated with safety climate such as working under conditions of clutter, extreme weather, and low visibility. These antecedents can be prevented by safety climate enhancements such as contingency planning to address poor working conditions and safety meetings to communicate the need for caution due to work conditions. In addition, limited resources in terms of equipment as well as time and human resources seemed to indicate the presence of stress as an antecedent to critical incidents.

A brief safety climate measure using job satisfaction, perceived vulnerability (likelihood of injury), and number of accidents experienced in the past year showed no significant associations with the exception of perceived vulnerability and accidents. Workers who had more accidents were more likely to rate their perceptions of likelihood of having an accident in the future higher as well. This finding has implications for future studies. Although no cause and effect can be concluded, the results may indicate that workers with more accidents could begin to believe accidents are more likely to occur in the future. Whether this leads to more careful behavior or more stress (and thus accidents), studies on attitude changes after accidents are important to pursue in future studies.

The cluster analysis results for the task or work patterns were not as clearly defined. In spite of the five-cluster solution, the predominant tasks performed when CIs occurred could be summarized into three patterns; roofing tasks, material handling, and machine operation and maintenance. These results may be helpful to prioritize training programs, and may alert foremen and supervisors to provide more oversight during performance of these tasks. Foreknowledge of the most hazardous tasks can support efficient functioning during a building project even when challenged by limited time and resources.

Although a useful number of CIs were acquired (102) from 14 workers, this amount is likely an underestimation of the actual number occurring during the observation period. Yet, the sample was large enough to document some of the problems that occur in small construction contexts. The goal of qualitative research methods is to achieve transferability [30], which is determined by the usefulness of the results as determined by other researchers examining the same construction situation or context.

Some of the limitations of the study were related to the use of critical incident logging, which was somewhat onerous to participants and thus had the potential to impact the reliability and validity of the information. While workers accepted the method and found it to be efficient, some reported experiencing problems recalling incidents if several occurred in the same day. The critical incident technique embedded within this context showed several of the same advantages and disadvantages found in Flanagan's method. Reporting of critical incidents soon after they occurred resulted in data that is less confounded by memory compared to the use of retrospective interview or questionnaire methods. In addition, critical incident reporting provides a descriptive $\log$ that is directly reported by the worker within their natural environment, which reduces the likelihood of diminished validity due to the use of artificial measurement instruments. We modified the technique by eliminating the direct observation component and relying only on workers' self-reported observations. This lower intrusiveness by the researcher minimizes demand effects. Direct observation is time-consuming and expensive, so tradeoffs were made based on the limited resources available to both the researchers and the participants. Use of the logging approach also provides the same advantage as that found in the narrative inquiry approach, such as allowing workers to report information in their own words using an anecdotal or storytelling voice rather than a formalized report that may interfere with how workers organize their memories around specific events or occurrences [31]. However, disadvantages or limitations are still a challenge to the collection of data for critical incidents. For example, workers need to be reminded to record incidents over the entire logging interval. This can be challenging to workers who have demanding workloads and are 
too fatigued to record after the work day has ended. Thus, the reliability and clarity of reporting must be improved.

We were not able to recruit as many participants as originally planned. Some workers initially committed to the project, but could not be located when it was time to begin data collection. The transient nature of construction workers makes it difficult to sustain a sufficiently large sample. We tried to recruit a small sample that would provide enough cases to identify general patterns. Typically, the qualitative practice is to sample until no new insights of the phenomenon emerge. Important factors to include in future studies involving the identification of construction safety critical incidents include the use of a more diverse sample in terms of ethnicity and construction trades and minimizing selfselection bias. The participants in this study were ethnically homogenous (European-American); thus the lack of diversity in the sample might have impacted the types of critical incidents reported and the patterns or clusters we identified. Self-selection bias, although it cannot be measured, likely influenced some of the data in this study. Workers who volunteered were highly motivated and tended to have concerns about safety in general. In spite of these challenges, the critical incident method has shown some promise as an assessment and surveillance tool in the small construction industry.

\section{ACKNOWLEDGEMENT}

This research was funded by the National Institute for Occupational Safety and Health (NIOSH), grant number OH008308 (Center for Innovation in Construction Safety and Health). Its contents are the authors' sole responsibility and do not necessarily represent official NIOSH views.

\section{REFERENCES}

[1] Bureau of Labor Statistics, US Department of Labor. (2006). Occupational Outlook Handbook, 2006 - 2007 Ed. [cited on 30 Apr 2009]. Available from: http://www.bls.gov/oco/

[2] Lin J, Mills A. Measuring the occupational health and safety performance of construction companies in Australia. Facilities 2001; 18: 131-9.

[3] Mlinga R, Wells J. Collaboration between informal and formal enterprises in the construction sector in Tanzania. Habitat Int 2002; 26: $269-80$

[4] Misra N. Policy Brief: The push and pull of globalization: How the global economy makes migrant workers vulnerable to exploitation. [cited 2009 Apr 20]. Available from: http://www.solidaritycenter. rg/files/pubs_policybrief_migration.pdf

[5] Bureau of Labor Statistics. National Census of Fatal Occupational Injuries in 2007. USDL-08-1182, August, 2008. [cited $30 \mathrm{Apr}$ 2009]. Available from: http://www.bls.gov/news.release/pdf/cfoi. pdf

[6] Garber R. Closing the Gap: Information Models in Contemporary Design Practice: Architectural Design. New York: John Wiley and Sons 2009.

[7] Uher T. Risks in subcontracting: subcontract conditions. Constr Manage Econ 1991; 9: 495-508.

[8] Mayhew C, Quinlan M, Ferris R. The effects of subcontracting/outsourcing on occupational health and safety: Survey evidence from four Australian industries. Saf Sci 1997; 25: 163-75.
[9] Browning LD, Beyer JM, Sheltler JC. Building cooperation in a competitive industry: SEMATECH and the semiconductor industry. Acad Manage J1995; 38: 113-51.

[10] Miller NJ, Besser T, Malshe A. Strategic networking among small businesses in small U.S. communities. Int Small Bus J 2007; 25: 651-65.

[11] Chua J, Chrisman J, Sharma P. Defining the family business by behavior. Entrep Theory Pract 1999; 23: 19-39.

[12] Marshall J, Sorenson R, Brigham K, Wieling E, Reifman A, Wampler R. The paradox for the family firm CEO: Owner age relationship to succession-related processes and plans. J Bus Venturing 2006; 21: 348-68.

[13] Davis G. Job satisfaction survey among employers in small businesses. J Small Bus Enterp Dev 2004; 11: 495-503.

[14] Chandler EN, Keller C, Lym DW. Unraveling the determinants and consequences of innovation supportive organizational culture. Entrep Theory Pract 2000; 25: 59-76.

[15] Gray JH, Densten IL, Surrow JC. Size matters: Organisational culture in small, medium, and large Australian organizations. Proceedings of the Administrative Sciences Association of Canada Conference 2003: 14-7; Halifax, Nova Scotia.

[16] Man TW, Lau T, Chan F. The competitiveness of small and medium enterprises: A conceptualization with focus on entrepreneurial competencies. J Bus Venturing 2001; 17: 123-42.

[17] Cooper MD. Towards a model of safety culture. Saf Sci 2000; 36:111-36

[18] Wiegmann DA, Zhang H, Von Thaden TL, Sharma G, Gibbons AM. Safety culture: an integrative review. Int J Aviation Psychol 2004; 14(2); 117-34.

[19] Flanagan J. The critical incident technique. Psychol Bull 1954; 51: 327-59.

[20] De Saram D, Ahmed S, Anon, M. Suitability of the CIT to measure quality of construction coordination. J Manage Eng 2004; 20: 97109.

[21] Marshall M. Sampling for qualitative research. Fam Pract 1996; 13 : 522-5.

[22] Patton M. Qualitative evaluation and research methods. $2^{\text {nd }}$ ed. Newbury Park: Sage 1990.

[23] Wiegmann DA, Zhang H, Von Thaden TL, Sharma G, Mitchell A. A synthesis of safety culture and safety climate research. Technical Report-Aviation Research Lab-02-3/FAA-02-2. University of Illinois-Urbana-Champagne.

[24] Sokal R, Sneath, P. Principles of numerical taxonomy. San Francisco: Freeman 1963.

[25] Bradley C. Turning anecdotes into data - the critical incident technique. Fam Pract 1992; 9: 98-103.

[26] Smith-Jackson TL, Wogalter MS, Quintela Y. Cultural ergonomics and the pesticide risk divide. Hum Factors Ergon Manuf Serv Indus 2010; 20: 511-25.

[27] Young B, Hall G. Factors associated with insolvency amongst contractors in the construction industry. Build Res Inf 1991; 9: 315-8.

[28] Champoux D, Brun, J-P. Occupational health and safety management in small size enterprises: An overview of the situation and avenues for intervention research. Saf Sci 2003; 41:301-18.

[29] Kotey B, Folker, C. Employee training in SMEs: Effect of size and firm type - family and nonfamily. J Small Bus Manage 2007; 45: 214-38.

[30] Hoepfl M. Choosing qualitative research: A primer for technology education researchers. J Technol Educ 1997; 9: 47-63.

[31] Clandinin D, Connelly M. Narrative inquiry: experience and story in qualitative research. San Francisco: Jossey-Bass Publishers 2000 .

() Smith-Jackson et al.; Licensee Bentham Open.

This is an open access article licensed under the terms of the Creative Commons Attribution Non-Commercial License (http://creativecommons.org/licenses/by-nc/3.0/) which permits unrestricted, non-commercial use, distribution and reproduction in any medium, provided the work is properly cited. 